\title{
INVESTIGATION OF ENERGY DISSIPATION IN LOW FREQUENCY VIBRATORY MEMS DEMONSTRATING A RESONATOR WITH 25 MINUTES TIME CONSTANT
}

\author{
S.A. Zotov, B.R. Simon, G. Sharma, J. Han, I.P. Prikhodko, A.A. Trusov, A.M. Shkel \\ MicroSystems Laboratory, University of California, Irvine, CA, USA
}

\begin{abstract}
We report a conventionally batch micromachined silicon tuning fork MEMS resonator, with ultra-low energy dissipation. The dissipation time constant of 25 minutes was experimentally achieved by using a test device with natural frequency of $570 \mathrm{~Hz}$ and Quality factor of 2.7 million. This low level of energy dissipation was accomplished through identification and minimization of the dominating dissipation mechanisms. Results of the experimental investigation lead to the formulation of practical design guidelines and tradeoffs for low frequency resonant devices.
\end{abstract}

\section{INTRODUCTION}

Dissipation of energy is a fundamental characteristic of a resonance system which defines a limit of performance. For some devices, such as 1-D oscillators, frequency multiplied by Q-factor, $\mathrm{f} \times \mathrm{Q}$, is the figure of merit [1]. For others, such as Coriolis Vibratory Gyroscopes (CVG), the dissipation time constant, $\tau$, is a parameter of interest. Macro-scale CVGs with size on the order of several inches can achieve a dissipation time constant of tens of minutes and Q-factor in the tens of millions, with bias instability on the order of $10^{-5} \mathrm{deg} / \mathrm{hr}$ [2]. Additionally, a high dissipation time constant provides advantageous mechanizations, such as continuous whole angle operation uninterrupted by power failures (i.e., electromagnetic impulse), which is not possible using optical devices, such as Fiber Optic Gyroscopes (FOGs) and Ring Laser Gyroscopes (RLGs).

Low dissipation vibratory systems, especially systems with a high time constant, are generally associated with large spatial dimensions. It is often assumed that conventionally batch fabricated silicon MEMS resonators are not suitable for applications where large dissipation time is necessary due to their small mobile masses. However, state of the art MEMS devices have demonstrated time constants up to three minutes in recent reports, [3], [4].

Unlike mature RF resonators, there is a gap in the literature in the field of low dissipation micromachined vibratory gyroscopes. In this paper, an ultra-low dissipation resonator is reported and used in an experimental study of design trade-offs and formulation of practical design guidelines. The fabricated prototype has demonstrated a time constant of $\sim 1 / 2$ hour and Q-factor of 2.7 million at a natural frequency of $570 \mathrm{~Hz}$, Fig. 1, as well as a high dissipation time constant and high value of $\mathrm{Q}$-factor throughout a wide temperature range, from $-40{ }^{\circ} \mathrm{C}$ to $100^{\circ} \mathrm{C}$.

For low frequency resonators there are five primary energy loss mechanisms. In order of importance, these are: viscous damping, support (anchor) loss, loss due to structural asymmetry, ThermoElastic Damping (TED), and electrical damping. These mechanisms comprise the total Q-factor of the device [5]:

$$
\frac{1}{Q_{\text {total }}}=\frac{1}{Q_{\text {Viscous }}}+\frac{1}{Q_{\text {Anchor }}}+\frac{1}{Q_{\text {Asym }}}+\frac{1}{Q_{T E D}}+\frac{1}{Q_{E l}}+\frac{1}{Q_{\text {Other }}} \text {. }
$$

Throughout this paper, each of these five dissipation mechanisms are systematically investigated, and the impact of each is quantitatively assessed. It is also experimentally validated that any additional damping mechanisms, Qother, are negligibly small for low frequency resonators.

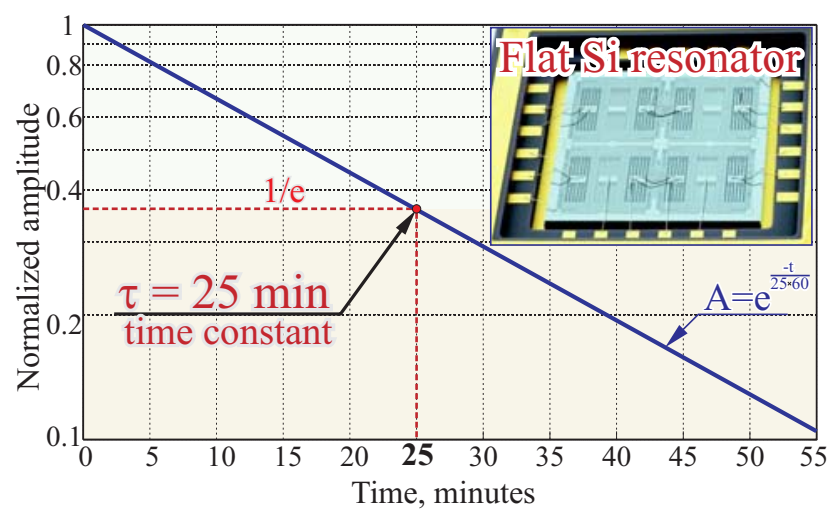

Figure 1: Experimental characterization of vacuum-sealed Si resonator using the amplitude decay method, revealing a dissipation time constant of 25 minutes (Q-factor of 2.7 million at $570 \mathrm{~Hz}$ natural frequency).

\section{TEST-BED RESONATOR}

To support this theoretical investigation, a specially designed resonator was fabricated. This resonator was designed to minimize energy dissipation from viscous damping, thermoelastic damping, and support loss. With these three mechanisms sufficiently reduced, a detailed investigation into electrical damping of the detection system is conducted.

To alleviate support losses, a tuning fork architecture was employed. The proposed tuning fork resonator comprises of two coupled tines ( $4 \mathrm{~mm}$ by $4 \mathrm{~mm}$ each), each driven in opposite directions, or anti-phase resonance. Each tine includes differential lateral comb electrodes for capacitive detection, and differential lateral comb electrodes for electrostatic excitation of the anti-phase mode. The anti-phase mode of resonator minimizes the net reaction force applied to the substrate, providing both rejection of commonmode external accelerations, and reduction of energy dissipation from the vibrating structure. Any in-phase component of motion is due to fabrication imperfection and is considered parasitic; to reduce undesirable in-phase motion the design includes nondifferential parallel plate capacitors for dynamic balancing by modulation of stiffness through the negative electrostatic spring effect, Fig. 2. Also, the parallel plate electrodes allow for the simultaneous and independent tuning of operational (resonance) frequency and Q-factor through electrical damping. The fabrication of the prototype was performed using an in-house, wafer-level, single-mask process. Devices were fabricated using Silicon-onInsulator (SOI) wafers with a $100 \mu \mathrm{m}$ single crystalline silicon device layer, a $5 \mu \mathrm{m}$ buried oxide layer, and a $500 \mu \mathrm{m}$ handle wafer, Fig. 2. After wafer fabrication and dicing, sensors were attached to a ceramic DIP-24 package through the use of a UniTemp RSS-160 solder reflow system, and a low-stress die attachment procedure. The attachment was made using an eutectic solder comprised of $80 \%$ gold and $20 \%$ tin. Eutectic $\mathrm{Au} / \mathrm{Sn}$ solder was used in this process for three main reasons: 1) it is a low outgassing material, well suited for vacuum sealing with getter, 2) it creates a strong, rigid attachment to the package, preventing spurious degrees of freedom, and 3 ) it is capable of surviving high temperatures. Due to mismatches in the thermal properties between 


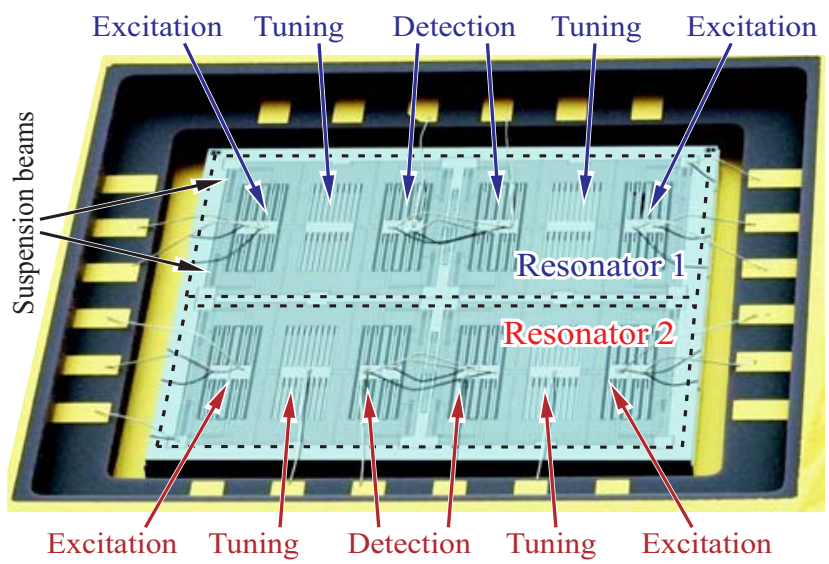

Figure 2: Optical photograph of the test-bed resonator, before vacuum sealing with getter material.

the package and die, changes in temperature can induce stress within the die, creating structural asymmetry and increasing the dissipation of energy through the substrate, [4]. This effect is minimized by reducing the die attachment area, as described in [6].

We demonstrated a tuning fork design with a time constant of 2 minutes, in [4]. The prototype was fabricated without reduction of the die attachment area, leading to energy dissipation through the substrate. Evidence is shown in Fig. 5a, where electrical tuning is able to increase the Q-factor of the device by increasing the structural symmetry, [4]. In contrast, the design of the tuning fork proposed in this paper took full advantage of the reduction of the die attachment area. As shown in Fig. 5b, any tuning voltage applied to the resonant masses increase the asymmetry, resulting in a reduction of $\mathrm{Q}$-factor.

\section{VISCOUS DAMPING}

Viscous damping is a first major contributor to energy loss in MEMS devices, especially for low frequency resonators. For the tuning fork demonstrated in this work, the device has a Q-factor of 4 in air, before vacuum sealing. The first step to raising the Qfactor is to provide vacuum, and ensure a stable pressure environment through the life of the sensor. To achieve this, the package of the device was sealed under high vacuum ( $1 \mu$ Torr). A thin-film getter material was sealed inside the packaged cavity in order to absorb any residual gases that may be present after the vacuum packaging process, compensating for out-gassing.

Glass lids were prepared using 4 inch wafers of D263 glass, specifically chosen to match the coefficient of thermal expansion of the ceramic package, $7.1 \mathrm{ppm} / \mathrm{K}$. These wafers were patterned with a thin layer of chrome and gold, $50 \mathrm{~nm}$ and $500 \mathrm{~nm}$, respectively.

The vacuum-sealing process was completed using a model 3150 , high vacuum furnace from SST International. Before sealing, a solder preform in the shape of a frame was tacked to each package at the interface of package and lid. The material of the solder frame was identical to the die attachment process: 80/20 $\mathrm{Au} / \mathrm{Sn}$ solder. Both package and lid were then separately loaded into the vacuum furnace, and vacuum sealed after several hours of prebaking, using the procedure as described in [6].

\section{THERMOELASTIC DAMPING}

Thermoelastic damping is a fundamental source of damping for any vibratory structure. It is a material-dependent loss mechanism that arises from the coupling of internal stress to the thermal domain through the coefficient of thermal expansion.

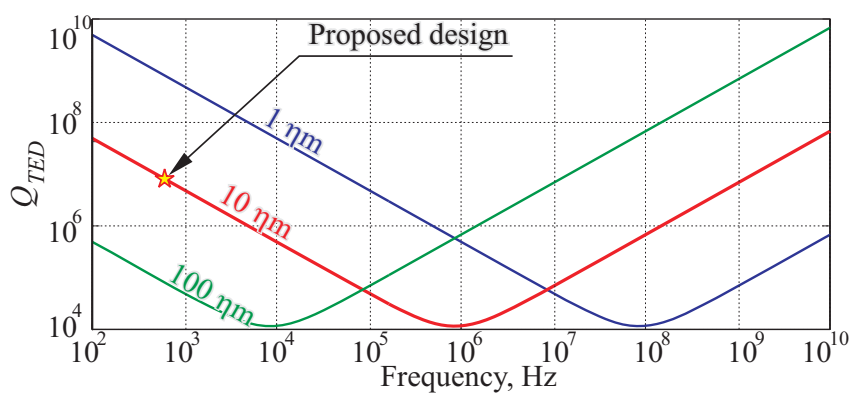

Figure 3: Thermoelastic Q-factor versus frequency for silicon springs of three different widths $(1,10$, and $100 \mu \mathrm{m})$.

Vibrations of flexing suspension elements create a stress gradient across its cross-section, which in turn induces a thermal gradient.

After this thermal gradient is equalized, resonant energy is reduced in the system, converting to entropy, [5]. Given a suspension beam that is experiencing sinusoidal flexing, energy loss is maximized (or Q-factor minimized), when choosing a mechanical resonance frequency with period that matches the time required for energy to flow from one side of the suspension beam to the other. This phenomenon can be analytically described by Zener's equation [7], which is plotted in Fig. 3 for silicon beams of various widths $(1-100 \mu \mathrm{m})$. Through choice of beam width, a trade-off exists between thermoelastic damping and structural asymmetry; thin beams increase thermoelastic Q-factor, but reduce structural symmetry, which directly influences losses through the substrate, as detailed in the following sections. In this work, a suspension beam element of $10 \mu \mathrm{m}$ in width is used.

The mechanical frequency that maximizes thermoelastic energy loss for beam widths of $10 \mu \mathrm{m}$ for silicon is roughly $900 \mathrm{kHz}$, Fig. 3. To minimize the thermoelastic damping effect, the period of the mechanical resonance must be separated from the thermal period as far as possible. This can be accomplished by changing the material or physical dimensions of the resonator.

While the above model is valid for a simple beam, it can only serve as an approximation for complex structures. For this reason, finite element modeling was conducted for the design using COMSOL Multiphysics, revealing a thermoelastic Q-factor of 5.7 million at a low operational frequency of $570 \mathrm{~Hz}$.

\section{SUPPORT LOSS}

Support loss, or anchor loss, is a form of energy loss due to stress waves propagating away from the resonant structure, though the anchors and into the substrate and package of the device. Designing devices that consist of two or more separate proof masses operating in an anti-phase mode shape is one method of reducing this effect, assuring the effective reaction forces to be equal to zero. While this can greatly reduce the effects of support loss, it does not eliminate it completely.

To gauge the effects of anchor loss for the given design, a 3-D FEA model was created containing the device, substrate, and a Perfectly Matched Layer (PML). The PML represents an infinite boundary, behaving as an acoustic absorption layer. Acoustic waves that enter the PML attenuate before they can be reflected back into the model, Fig. 4(a). The footprint of the substrate is 4.1 $\mathrm{x} 8.1 \mathrm{~mm}$. In this study, a PML beneath the substrate is chosen to absorb one wavelength of stress, transmitted at the resonant frequency of the vibratory modes. The Q-factor of the anti-phase mode is calculated to be $Q_{\text {Anchor }}=5.67 \times 10^{6}$. Mesh elements, varied from $114 \times 10^{3}$ to $218 \times 10^{3}$, confirm the convergence of the model with a tolerance of $1 \%$, Fig. 4 (b). 


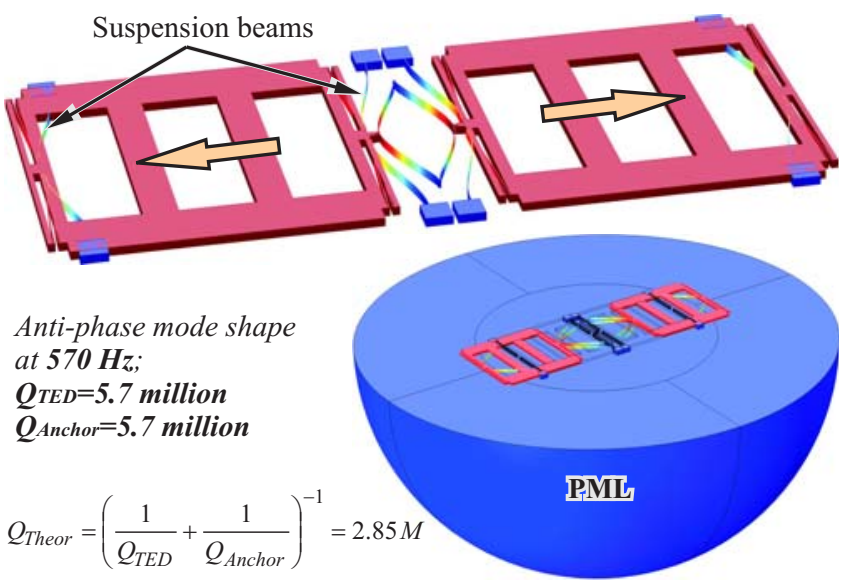

a) 3-D anchor loss model, highlighting tuning fork and PML layer.

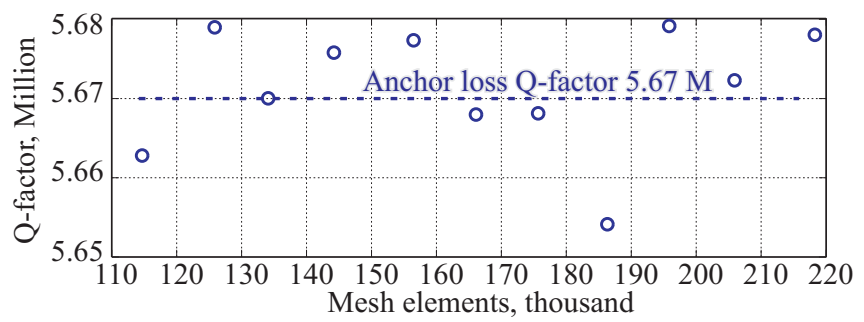

b) Dependence of Q-factor for anti-phase mode on the number of elements.

Figure 4: Finite element modeling of energy dissipation through the substrate (anchor loss) reveals a Q-factor of the anti-phase mode of $5.67 \times 10^{6}$, illustrating the theoretical Q-factor limit of 2.85 million for the anti-phase vibratory mode of the tuning fork.

\section{LOSS DUE TO STRUCTURAL ASYMMETRY}

In the previous section, the calculated support loss is for an ideal anti-phase vibratory mode. While this result offers the minimum support loss, it does not account for any fabrication imperfections within the structure that create variances in the nominal mass and stiffness values. Any asymmetry between the two resonant masses has the potential to induce additional energy loss through the anchor due to unbalanced motion. Despite driving the resonator in anti-phase motion, this structural asymmetry couples the anti-phase motion into in-phase motion. This mechanism, as well as a technique of dynamically balancing tuning forks to alleviate substrate losses, is theoretically and experimentally studied in [4].

A voltage applied to the tuning electrode of each tine was used to tune the dissipation time constant and quality factor of the resonator by disturbing the dynamic balance of the structure, Fig 5 . While the design presented previously in [4] required additional tuning to achieve dynamic balance, Fig $5 \mathrm{a}$, the proposed design is initially well balanced, Fig 5b. For the design studied in this paper, the experiment reveals that any tuning of stiffness in the left or right tines results in additional misbalance, reducing the Q-factor and dissipation time constant, Fig 5b.

\section{ELECTRICAL DAMPING}

Electrical damping can occur as a result of interference between electrical components when using electrostatic detection. Usually, this affect is minimal, which allows other energy loss mechanisms to dominate. However, for low dissipation resonators, it can be beneficial for independent tuning of Q-factor. Existing methods of Q-factor tuning couple inseparably Q-factor and

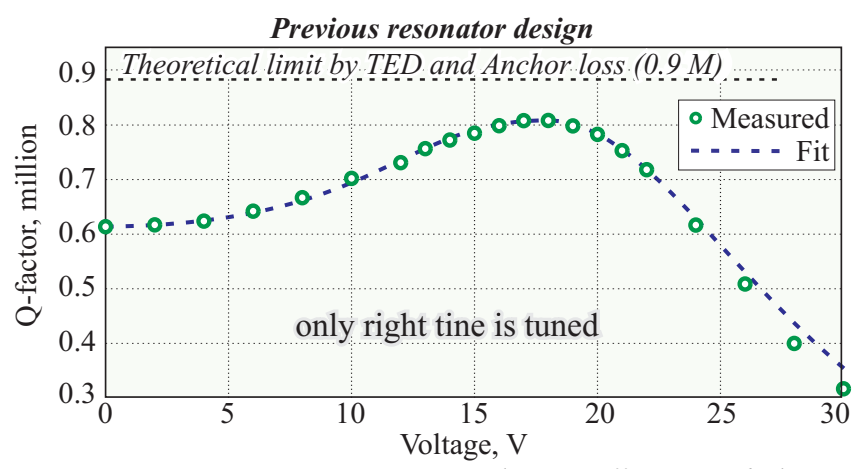

a) First generation resonator reveals a stiffness misbalance between the right and left tines of $1.5 \%$ before tuning (see [4] for details).

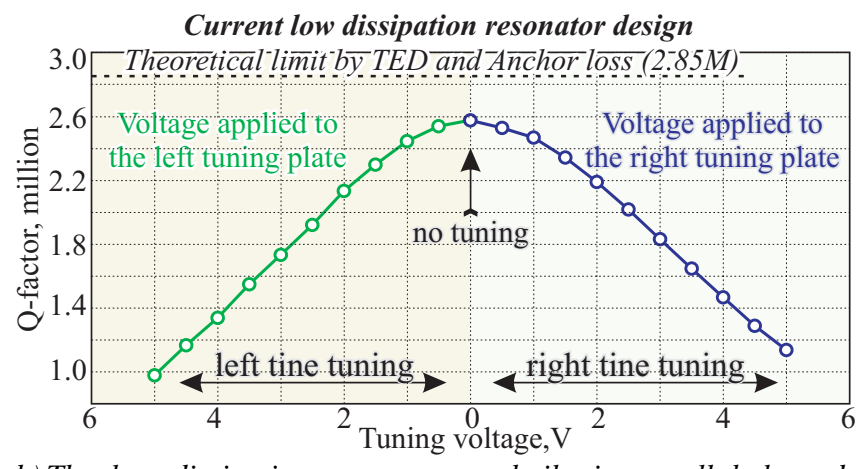

b) The low dissipation resonator, as-built, is a well balanced device, measured with a precision of less than $20 \mathrm{ppm}$.

Figure 5: Characterization of the stiffness matching effect on the resonator's Q-factor by measuring $Q$-factor of the anti-phase mode versus tuning voltage applied to the left and right tines.

frequency through the use of an electrostatic spring, inducing a momentum imbalance [4]. In contrast, when electrical damping is introduced, only the Q-factor is affected. This technique is useful for gyroscopes, especially when operated in whole-angle mode, where independent tuning of frequency and Q-factor is critical. However, this phenomenon imposes additional restrictions on the parameters of the detection system, specifically the electrical gain of the detection pick-off. A trade-off exists between the pick-off gain and Q-factor tuning ability, which is experimentally demonstrated in this section.

To demonstrate the phenomena, the packaged resonator was mounted on a two stage PCB, Fig. 6. The top stage of the electronics is comprised of front-end transimpedance amplifiers, while the bottom stage stabilizes input voltages and creates the device excitation signals. Electrostatic actuation and capacitive detection were employed along with the ElectroMechanical Amplitude Modulation (EAM) technique to eliminate parasitic feed-through. Instead of a DC bias, an AC carrier voltage with variable amplitude at $52 \mathrm{kHz}$ frequency was applied to the mobile masses. The anchored differential sense-mode comb-electrodes were connected to the inputs of a two-stage differential transimpedance amplification circuit implemented on the PCB, Fig. 6. All signal processing is performed in real-time using an FPGA-based lock-in amplifier from Zurich Instruments.

To show the influence of the detection system on the Q-factor of the tuning fork, Q-factor was measured versus carrier amplitude from $0.3 \mathrm{~V}$ to $6 \mathrm{~V}$ for four different feedback resistors $(24,46,91$, and $196 \mathrm{kOhm}$ ), along with feedback capacitors of $10 \mathrm{pF}$. The experimental results demonstrate that Q-factor is reduced as carrier 


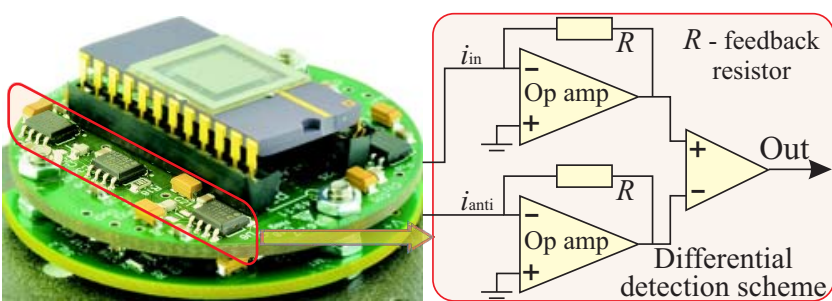

Figure 6: Photograph of a packaged differential tuning fork assembled with signal conditioning PCBs, realized with signal transimpedance detection. The feedback capacitor is not shown.

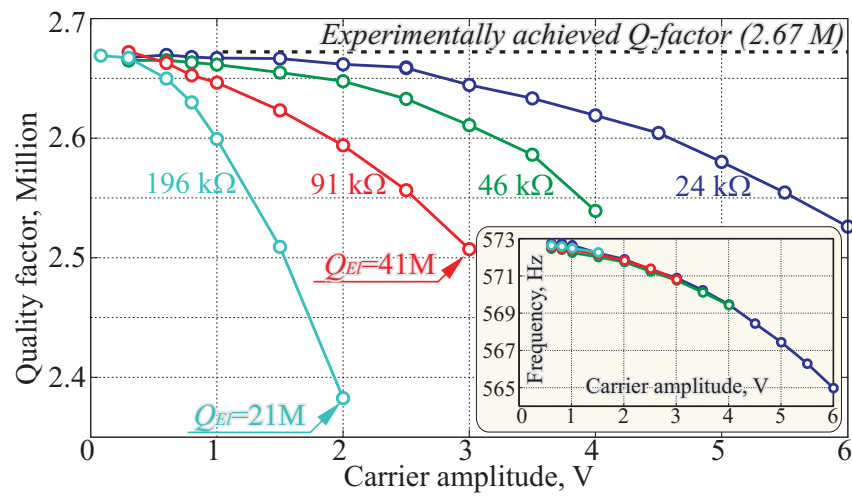

Figure 7: Experimentally measured Q-factor vs. carrier amplitude for various feedback resistors. Q-factor lowers as carrier amplitude increases. (Inset: natural frequency vs. carrier amplitude for the same experiment).

amplitude is increased, and this effect is more pronounced for large values of feedback resistance. Considering feedback resistance is directly correlated to electronic gain, increasing this gain has the potential to be a limiting mechanism of Q-factor. This effect is demonstrated in Fig. 7, which also displays the natural frequency of the resonator for each data point. While the natural frequency is influenced by the amplitude of the carrier voltage, there is no influence from changes in the feedback resistance. By using formula (1), electrical Q-factor, $Q_{E l}$, was estimated for different feedback resistors, R, and carrier voltage, V. For $\mathrm{R}=196 \mathrm{kOhm}$ and $\mathrm{V}=2$ Volts, $Q_{E I}=22$ million. For $\mathrm{R}=91 \mathrm{kOhm}$ and $\mathrm{V}=3$ Volts, $Q_{E l .}=41$ million, Fig. 7. This phenomenon provides a mechanism for an independent tuning of frequency and Q-factor.

Characterization of Q-factor and dissipation time constant at different temperatures, over a range from $-40{ }^{\circ} \mathrm{C}$ to $+100{ }^{\circ} \mathrm{C}$, reveals a time constant of over 14 minutes (Q-factor $=1.65$ million), with maximum value of over 25 minutes (Q-factor $=2.67$ million) for temperatures below $20^{\circ} \mathrm{C}$, Fig. 8. In addition, using the same balancing technique as described in section "Loss due to structural asymmetry" and in [4], it was confirmed that across various temperatures, ranging from $-40{ }^{\circ} \mathrm{C}$ to $+100{ }^{\circ} \mathrm{C}$, the resonator remained dynamically balanced.

The experimentally measured maximum Q-factor of 2.67 million was achieved using a low electrical dissipation setup of a $24 \mathrm{k} \Omega$ feedback resistor and carrier amplitude of 0.3 Volts, Fig. 7. At the same time, the contribution of both TED and anchor loss equally limit the Q-factor to 5.7 million, providing the theoretical limit of 2.85 million, which is $9 \%$ larger than the experimentally measured value. This discrepancy can be attributed to additional, unaccounted for damping mechanisms: residual viscous damping, electrical damping, surface loss, loss due to structural asymmetry, and other energy loss mechanisms, described in [5]. Using formula (1), with $Q_{\text {total }}=2.67$ million and $Q_{a n c h o r}=Q_{T E D}=5.7$ million, the unaccounted for Q-factor can be calculated to be 43 million.

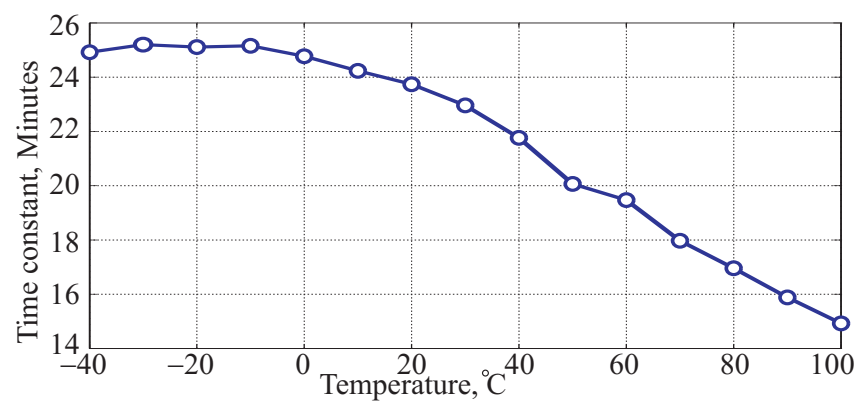

Figure 8: Measured dissipation time constraint versus temperature for a vacuum sealed tuning fork resonator.

\section{CONCLUSION}

This paper presents a conventionally batch-fabricated, vacuum-packaged, silicon MEMS resonant device with a time constant of 25 minutes and a quality factor of 2.67 million at a natural frequency of $570 \mathrm{~Hz}$. The reported time constant is comparable to that of the macro-scale hemispherical resonator gyroscope, bridging the gap between flat MEMS and 3-D quartz devices. The resonator demonstrates a high dissipation time constant and a high Q-factor throughout a wide temperature range, from $-40{ }^{\circ} \mathrm{C}$ to $+100{ }^{\circ} \mathrm{C}$. These results are achieved by specifically targeting and minimizing influences of the five primary sources of damping: viscous damping, support (anchor) loss, loss due to structural asymmetry, ThermoElastic Damping (TED), and electrical damping. We also experimentally validated that the detection system can be a measurable source of energy dissipation. Finally, we demonstrated that the Q-factor can be tuned independently of other system parameters, such as frequency.

\section{ACKNOWLEDGMENT}

This work was supported by DARPA under grant N66001-12C-4035. Authors would like to thank Dr. Flavio Heer of Zurich Instruments AG as well as David Muhs, Pierino Zappella of SST International. The devices were designed and tested at the MicroSystems Laboratory, UC Irvine.

\section{REFERENCES}

[1] J.E-Y. Lee and A.A. Seshia, "Square wine-glass mode resonator with a Q-Factor of 4 million," IEEE Sensors, Lecce, Italy, 2008, pp. $1257-1260$.

[2] D.M. Rozelle, "The hemispherical resonator gyro: from wineglass to the planets," in: Proc. of the 19th AAS/AIAA Space Flight Mechanics Meeting, Feb. 2009, pp. 1157 - 1178.

[3] S.A. Zotov, A.A. Trusov, and A.M. Shkel, "Experimental demonstration of a wide dynamic range angular rate sensor based on frequency modulation," IEEE Sensors, Limerick, Ireland, 2011, pp. $577-580$.

[4] A.A. Trusov, S.A. Zotov, and A.M. Shkel, "Electrostatic Regulation of Quality Factor in Tuning Fork MEMS," IEEE Sensors, Limerick, Ireland, 2011, pp. 20 - 23.

[5] V.B. Braginsky, V.P. Mitrofanov, and V.I. Panov, "Systems with small dissipation," Univ. Chicago Press, 1985, p. 145.

[6] I.P. Prikhodko, B.R. Simon, G. Sharma, S.A. Zotov, A.A. Trusov, and A.M. Shkel, "High and Moderate Level Vacuum Packaging of Vibratory MEMS," IMAPS, Orlando, FL, 2013, pp. $705-710$.

[7] C. Zener, "Internal friction in solids I: Theory of internal friction in reeds," Phys. Rev., vol. 52, no. 3, pp. 230 - 235.

\section{CONTACT}

*S.A. Zotov, email: zotov.sergei@gmail.com 